Recepción: 28 / 03 / 2017

Aceptación: 13 / 05 / 2017

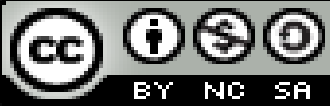

Ciencias técnicas y aplicadas

Publicación: 15 / 07 / 2017

Artículo de investigación

\title{
Evaluación de un calentador solar de agua de cilindro parabólico en la ciudad de Riobamba
}

\section{Evaluation of a parabolic-cylinder solar water heater in Riobamba city}

\section{Avaliação de um aquecedor de água solar com cilindro parabólico na cidade de}

\section{Riobamba}

Alexis L. Chamorro Páez ${ }^{\mathrm{I}}$ alexislcp@hotmail.es

Victor P. Arellano Mancheno II

paolo.arellano@yahoo.com

Marco A. Ordoñez-Viñán ${ }^{\text {III }}$ marco.ordonezo@espoch.edu.ec

Lenin S. Orozco-Cantos ${ }^{\mathrm{IV}}$

1sorozco@espoch.edu.ec

Edwin F. Viteri-Núñez ${ }^{\mathrm{V}}$

eviteri@espoch.edu.ec

Edwin A. Jácome-Domínguez VI

ejacome@espoch.edu.ec

Correspondencia: alexislcp@hotmail.es

'Ingeniero Mecánico, Docente de la Escuela Politécnica de Chimborazo, Riobamba, Ecuador.

"Ingeniero Mecánico, Docente de la Escuela Politécnica de Chimborazo, Riobamba, Ecuador.

"II Ingeniero Mecánico, Docente de la Escuela Politécnica de Chimborazo, Riobamba, Ecuador.

${ }^{\text {Iv }}$ Ingeniero Mecánico, Docente de la Escuela Politécnica de Chimborazo, Riobamba, Ecuador.

${ }^{\vee}$ Magister en Gerencia de Proyectos de Ecoturismo, Ingeniero Mecánico, Docente en la Escuela Politécnica de Chimborazo, Riobamba, Ecuador.

${ }^{\mathrm{V} 1}$ Ingeniero Mecánico, Docente de la Escuela Politécnica de Chimborazo, Riobamba, Ecuador. 


\section{Resumen}

En el presente trabajo se presenta la modelación matemática del perfil de temperaturas en el tubo absorbedor del concentrador solar cilíndrico parabólico en estado estacionario. La modelación matemática permite conocer el comportamiento de la ganancia de calor que experimenta el fluido en sentido longitudinal, en este caso agua, mientras permanece en reposo dentro del tubo absorbedor. Este modelado se logró a partir de un balance de energía en el tubo recibidor, que se encuentra en función de parámetros geométricos, ópticos y ambientales. Los resultados mostraron un perfil de temperaturas en estado estacionario que es de tipo exponencial. Se experimentó el tiempo de calentamiento de agua en el colector solar hasta los $70^{\circ} \mathrm{C}$, obteniéndose como resultado un tiempo aproximado de 7 minutos cuando se tiene el mayor índice de radiación, siendo la capacidad aproximada de 50 litros de agua caliente al día. Se analizó el calentamiento de agua a distintas velocidades, se obtuvo a que a una menor velocidad hay un mayor aumento en la temperatura. Se hizo una comparación entre un colector solar de tubos al vacío y el colector solar cilíndrico parabólico, siendo el primero mucho más eficiente, con un valor de $74 \%$ como la máxima eficiencia, mientras que en el colector cilíndrico parabólico se obtuvo una eficiencia máxima de $35,84 \%$. Esto es debido a que el tubo del primer colector por encontrarse al vacío reduce significativamente las pérdidas térmicas lo cual ayuda a mejorar la eficiencia.

Palabras clave: energías alternativas; calentador solar; cilíndrico parabólico; tubo absorbedor; reflector solar; sistema de seguimiento solar; modelación matemática.

\section{Abstract}

In the present work the mathematical modeling of the temperature profile in the absorber tube of the parabolic cylindrical solar concentrator in steady state is presented. The mathematical modeling allows to know the behavior of the heat gain that the fluid experiences in the longitudinal sense, in this case water, while remaining at rest inside the absorber tube. This model was achieved from an energy balance in the receiver tube, which is based on geometric, optical and environmental parameters. The results showed a steady-state temperature profile that is of exponential type. The water heating time in the solar collector was tested up to $70^{\circ} \mathrm{C}$, resulting in an approximate time of seven minutes approximately when there is the higher radiation index, being the approximate capacity 50 liters of hot water per day. The water heating was analyzed at different speeds. It was obtained that at a lower speed there is an increase in the temperature. A 
comparison was made between a solar collector of vacuum tubes and the parabolic cylindrical solar collector, the first one being much more efficient, with a value of $74 \%$ as the maximum efficiency, while in the parabolic cylindrical collector a maximum efficiency of $35.84 \%$. This is due to the fact that the tube of the first collector being vacuumed reduces significantly the thermal losses which helps to improve the efficiency.

Keywords: alternative energies; solar heater; parabolic cylindrical; absorber tube; solar reflector; sun tracking system; mathematic modeling.

\section{Resumo}

Neste documento, a modelação matemática do perfil da temperatura é apresentado no tubo absorvedor do estado estacionário concentrador solar parabico cildrico. A modelação matemática pode conhecer o comportamento de ganho de calor sofrida pelo fluido longitudinalmente, neste caso, água, mantendo-se em repouso no interior do tubo amortecedor. Esta modelação é obtida a partir de um balanço de energia no tubo receptor, que é uma função dos parâmetros geométricos, ópticos e ambientais. Os resultados mostraram um perfil de temperatura em estado estacionário é exponencial. o tempo de aquecimento da água no colector solar é experimentada a $70^{\circ} \mathrm{C}$, resultando num tempo de cerca de 7 minutos, quando tem a radiação mais alta, com a capacidade de cerca de 50 litros de água quente por dia. aquecimento de água foi analisada a velocidades diferentes, foi obtida a uma taxa mais lenta do que um maior aumento na temperatura. Foi feita uma comparação entre os tubos de vácuo de colector solar e o cilíndrico colector solar parabólico, sendo muito mais eficiente em primeiro lugar, com um valor de $74 \%$ como a eficiência máxima, enquanto o colector parabólico eficiência máxima cilíndrica foi obtido $35,84 \%$. Isto é porque o primeiro tubo colector para ser vácuo reduz significativamente a perda de calor que ajuda a melhorar a eficiência.

Palavras chave: energia alternativa; aquecedor solar; parabólica tubo; absorvedor cilíndrica; reflector solar; sistema de rastreamento solar; modelagem matemática.

\section{Introducción}

Los altos niveles de contaminación mundial producida en su mayoría por la quema de combustibles fósiles, han motivado al desarrollo de energías alternativas. Dentro de las energías alternativas más utilizadas está la energía solar, eólica, hidráulica, todas éstas provenientes de recursos renovables como: el sol, el viento y el agua respectivamente. En la ciudad de Riobamba 
las dos principales fuentes para calentar agua son: calefones con bombonas de propano y butano, y las duchas eléctricas. Los calefones emiten gases contaminantes a la atmósfera producto de la combustión, en cambio una ducha eléctrica posee una baja eficiencia. Parte de aquí la necesidad de desarrollar calentadores solares eficientes, seguros, y sobre todo de bajo impacto ambiental. La ciudad de Riobamba tiene un porcentaje promedio de radiación de 4,48 [kWh/m²], (NASA, 2016) debido a su ubicación geográfica junto al volcán Chimborazo el cual es el punto más cercano al sol. Esto hace que el uso de calentadores solares sea de mucha aplicación aquí en la ciudad obteniendo buenos resultados.

\section{Metodología}

\section{Cálculo del calor útil del sistema con el fluido estancado.}

El calor útil del sistema se obtiene mediante el cálculo de las pérdidas térmicas del sistema, mediante el coeficiente de pérdida $U_{L}$, el cual depende del área del receptor, y también mediante el factor de extracción de pérdida de calor $\mathrm{F}_{R}$

La pérdida de calor en el calentador va desde el tubo de cobre hacia la parte interna de la cubierta de vidrio, luego desde la parte exterior de la cubierta de vidrio hacia el ambiente; ésta pérdida de calor debe ser igual en ambos casos y se determinan por las siguientes expresiones:

$$
\begin{gathered}
Q_{\text {perdido } a-i v}=\frac{2 \pi k_{e f} L}{\ln \left(\frac{D_{i v}}{D_{e}}\right)}\left(T_{v}-T_{i v}\right)+\frac{\sigma A_{c}\left(T_{c}^{4}-T_{i v}^{4}\right)}{\frac{1}{s_{e}}+\frac{1-\varepsilon_{v}}{s_{v}}\left(\frac{D_{c}}{D_{i v}}\right)} \\
Q_{\text {perdido } i v-a m b}=h_{v} A_{v}\left(T_{e v}-T_{a m b}\right)+\varepsilon_{v} \sigma A_{v}\left(T_{e v}^{4}-T_{\text {cielo }}^{4}\right)
\end{gathered}
$$

Donde:

$Q_{\text {perdido } \mathbb{C}-\mathbb{L V}^{\mathrm{x}}}$ Calor perdido por convección y radiación desde el tubo receptor de cobre hacia la parte interior de la cubierta de vidrio. [W]

$Q_{\text {perdidoniv-ent }}{ }^{\frac{x}{x}} \quad$ Calor perdido por convección y radiación desde la parte externa del tubo de vidrio hacia el ambiente. [W]

$k_{\text {ef }}$ Conductividad térmica efectiva $[\mathrm{W} / \mathrm{Km}]$

$L_{\Sigma}$ Longitud del tubo de cobre $[\mathrm{m}]$ 
$T_{\mathrm{e}}$ : Temperatura de la tubería de cobre $\left[{ }^{\circ} \mathrm{K}\right]$

$T_{i v^{ \pm}}=$Temperatura de la parte interna de la cubierta de vidrio $\left[{ }^{\circ} \mathrm{K}\right]$

$D_{i w^{E}}$ Diámetro de la parte interna de la tubería de vidrio [m]

$D_{c}$ : Diámetro de la tubería de cobre $[\mathrm{m}]$

$\sigma_{\text {: }}$ Constante de Stefan-Boltzmann $\left[\frac{W}{m^{8} K^{4}}\right]$

$A_{\mathbb{Q}^{E}}$ Área de la tubería de cobre $\left[\mathrm{m}^{2}\right]$

$\varepsilon_{\mathrm{v}}:$ Emisividad de la cubierta de vidrio [adimensional]

$\varepsilon_{\mathbb{Q}^{\star}}$ Emisividad de la tubería de vidrio [adimensional]

$h_{\mathrm{v}^{ \pm}}$Coeficiente de transferencia de calor por convección debido al viento. [W/m² $\left.\mathrm{K}\right]$

Tev: Temperatura de la cubierta de vidrio $[\mathrm{K}]$

Tamb: Temperatura ambiente [K]

$T_{\text {cielo }}$ : Temperatura del cielo $[\mathrm{K}]$

Se asume un dato de temperatura de la parte exterior de la cubierta de vidrio ya que es un dato desconocido, esta estimación debe ser cercana a la temperatura ambiente. La temperatura del tubo de cobre es un dato conocido que fue medido mediante un pirómetro. Con el dato de temperatura de la cubierta de vidrio se resuelve la ecuación de las pérdidas de calor por convección y radiación desde la cubierta de vidrio hacia el ambiente, luego de esto se calcula las pérdidas de calor desde el tubo absorbedor de cobre hacia la parte interior de la cubierta de vidrio. Si ambos valores resultan iguales quiere decir que el dato asumido es correcto, de lo contrario se vuelve a asumir otro valor, con lo cual el proceso se vuelve iterativo.

Con la obtención de estos valores se calcula el coeficiente de pérdida $U_{L}$

$$
U_{L}=\frac{\left(Q_{\text {pardido }}\right)}{A_{E}\left(T_{e}-T_{a m b}\right)}
$$

Donde:

$U_{L^{E}}$ Coeficiente de pérdida

$A_{0^{E}}$ Área de la tubería de cobre $\left[\mathrm{m}^{2}\right]$

$T_{\mathbb{e}}$ : Temperatura de la tubería de cobre $\left[{ }^{\circ} \mathrm{K}\right]$ 
$T a m b$ Temperatura ambiente $[\mathrm{K}]$

Se calcula el factor de eficiencia del colector, este factor representa la relación entre ganancia de energía útil real y la ganancia de energía útil si el absorbedor estuviera a la temperatura de fluido local. Se obtiene mediante la siguiente expresión:

$$
F^{\prime}=\frac{1 / U_{L}}{\frac{1}{U_{L}}+\frac{D_{g \varepsilon}}{h_{f i} D_{i e}}+\left(\frac{D_{g e}}{2 k} \ln \left(\frac{D_{g \varepsilon}}{D_{i e}}\right)\right)}
$$

Donde:

$D_{\text {eqC }}$ : Diámetro externo de la tubería de cobre [m]

$D_{\text {ie }}$ : Diámetro interno de la tubería de cobre [m]

$\mathrm{h}_{\mathrm{fi}}:$ Coeficiente de transferencia de Calor dentro del tubo $\left[\mathrm{W} / \mathrm{m}^{2} \mathrm{~K}\right]$

El factor de remoción de calor del colector $F_{R}$ compara la cantidad de energía obtenida útil real del colector con la ganancia de energía útil si la superficie del colector estuviera a la temperatura de entrada del fluido, y debido a que el agua permanece estancada este valor es igual al factor de eficiencia del colector.

La ganancia de calor útil que se produce en el colector es igual a la radiación solar absorbida menos las pérdidas de calor que se producen en el colector.

$$
Q_{u}=F_{R} A_{a} R-A_{e} U_{L}\left(T_{e}-T_{a m b}\right)
$$

Donde:

$F_{R}:$ Factor de remoción de calor del colector $\left[\mathrm{Jm}^{2} / \mathrm{s} . \mathrm{W}^{2}\right]$

$A_{\mathbb{a}^{\circ}}$ Área de apertura del colector solar $\left[\mathrm{m}^{2}\right]$

$R \approx$ Radiación absorbida por unidad de área $\left[\mathrm{W} / \mathrm{m}^{2}\right]$

\section{Cálculo del tiempo de calentamiento de agua hasta $70^{\circ} \mathrm{C}$}

Una vez hallado el valor del calor útil generado por el colector solar se calcula el tiempo que se demora en calentar el fluido hasta la temperatura de $70^{\circ} \mathrm{C}$ mediante la siguiente expresión: 


$$
t=\frac{M}{Q_{u}} C_{p}\left(T_{s f}-T_{e f}\right)
$$

Donde:

tx Tiempo de calentamiento de fluido $[\mathrm{s}]$

$Q_{\mathrm{M}}:$ Calor útil generado por el colector solar $[\mathrm{W}]$

$M \approx$ Masa de fluido $[\mathrm{kg}]$

$C_{\mathrm{p}^{\mathrm{x}}}$ Calor especifico del fluido de transferencia de calor a presión constante $\left[\mathrm{J} / \mathrm{kg}^{\circ} \mathrm{K}\right]$

$T_{s f^{\star}}$ Temperatura a la salida del fluido $[\mathrm{K}]$

$T_{\text {eff }}$ Temperatura a la entrada del fluido $[\mathrm{K}]$

\section{Cálculo del incremento de temperatura del fluido cuando atraviesa el tubo absorbedor a diferentes velocidades.}

Para poder calcular este incremento en la temperatura se necesita el calor útil, la fórmula es la misma que la explicada en el punto 3.1., con la diferencia que el factor de remoción es igual a:

$$
F_{R}=F^{\prime} * F^{n}
$$

Donde:

$F^{n s}$ : Es el factor de flujo del colector y se determina por la siguiente expresión:

$$
F^{s y}=\frac{\dot{m} C_{p}}{A_{v} U_{L} F^{\prime}}\left(1-\exp \left(-\frac{A_{c} U_{L} F^{\prime}}{\dot{m} C_{p}}\right)\right)
$$

Para calcular el incremento de temperatura a diferentes velocidades de fluido se parte de la expresión de calor:

$$
Q_{u}=\dot{m} C_{p} \Delta T
$$

Donde:

$\underset{m}{m}$ Flujo másico $[\mathrm{Kg} / \mathrm{s}]$ 
Despejando la variación de temperatura:

$$
\Delta T=\frac{\dot{m} C_{\mathrm{p}}}{Q_{\mathrm{u}}}
$$

El flujo másico depende del valor de la velocidad, y éste a su vez del caudal que ingresa en la tubería de cobre.

\section{Eficiencia térmica del calentador solar cilíndrico parabólico}

La eficiencia de un concentrador solar se define como la relación entre la energía absorbida por el fluido portador del calor y la energía incidente que es la densidad de energía solar sobre su superficie.

$$
\eta_{c}=\frac{Q_{u t}}{I * A_{a}}
$$

\section{Modelación matemática que permite encontrar el perfil de temperaturas en sentido longitudinal del tubo absorbedor}

En el tubo absorbedor existe una ganancia térmica en sentido radial y longitudinal. El siguiente análisis se realiza para una ganancia de calor de forma longitudinal.

Para la modelación matemática se considera un elemento infinitesimal dx de la tubería de cobre, en este caso se obtendrá un perfil de temperaturas en sentido longitudinal del tubo absorbedor, por lo tanto se plantea las siguientes consideraciones:

- No se considera la distribución térmica en el área efectiva del sistema, ya que la mayor ganancia de calor se produce en sentido radial.

- $\quad$ No existe un cambio de fase dentro del fluido.

- $\quad$ Se trata de un sistema cerrado.

- $\quad$ Estado estacionario.

$\mathrm{Al}$ analizar el proceso en estado estacionario, se reduce significativamente el análisis de la transferencia de calor en el tubo receptor. 
Figura 5. Balance de energía en un elemento infinitesimal del tubo absorbedor

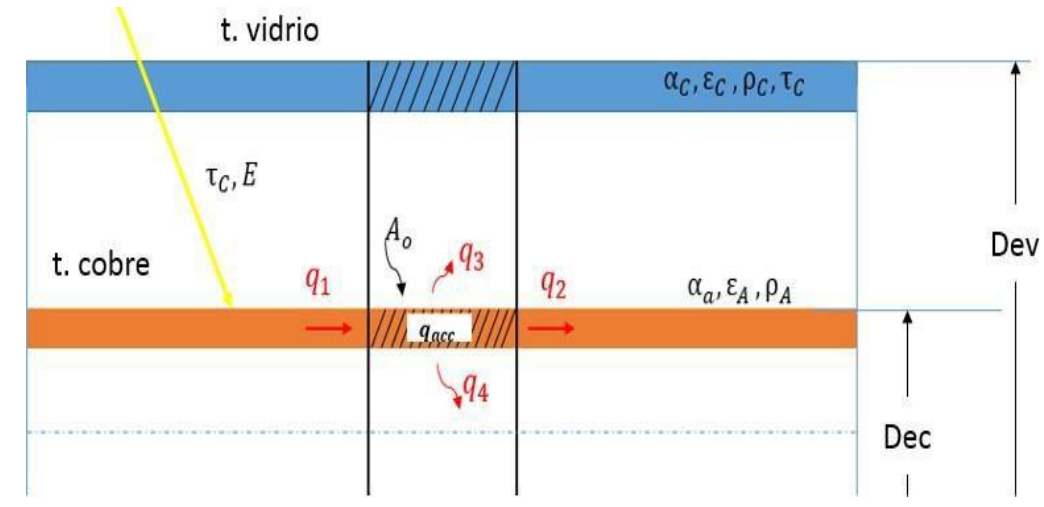

El calor que ingresa por conducción en el elemento infinitesimal dx viene dada por la siguiente expresión:

$$
Q_{1}=-k A_{\bullet} \frac{\partial T}{\partial x}
$$

El calor que sale por la sección $x+d x$ es igual a:

$$
Q_{2}=-k A_{\bullet} \frac{\partial T}{\partial x}+\frac{\partial}{\partial x}\left[-k A_{\bullet} \frac{\partial T}{\partial x}\right] d_{z}
$$

Existe calor por convección hacia el ambiente y hacia el fluido y se determina por las siguientes expresiones respectivamente:

$$
\begin{aligned}
& Q_{3}=U_{0} \pi D_{e v} d_{x}\left(T_{c}-T_{a m b}\right) \\
& Q_{4}=U_{i} \pi D_{i c} d_{s}\left(T_{c}-T_{i}\right)
\end{aligned}
$$

La ganancia de calor en el tubo absorbedor debido a la radiación solar incidente viene expresada por:

$$
Q_{s o l}=\rho \pi \alpha I \pi D_{c} d_{z}
$$

El calor acumulado dentro del elemento infinitesimal en el que se realiza el análisis está directamente relacionado con el aumento de temperatura y el calor especifico del material. Esta acumulación de calor se encuentra determinada por la siguiente expresión en función de la velocidad en la variación de temperatura: 


$$
Q_{\text {acu }}=\rho_{c} C_{p} A_{c} d_{x} \frac{\partial T}{\partial t}
$$

Realizando un balance de energía se determina que el calor que ingresa en el elemento infinitesimal menos el calor que sale es igual al calor acumulado en la masa de espesor $\mathrm{dx}$.

$Q_{B}-Q_{s}=Q_{\text {acu }}$

$$
Q_{1}+Q_{\text {sol }}-\left(Q_{2}+Q_{3}+Q_{4}\right)=Q_{\text {acu }}
$$

En este punto se hacen ciertas consideraciones para facilitar la resolución de la ecuación diferencial, llega un instante en el que se consigue un equilibrio térmico, por lo tanto el calor que recibe del sol sería el mismo que entra al fluido, tomando en cuenta lo dicho se llega a lo siguiente:

$$
k A_{\bullet} \frac{\partial^{2} T}{\partial x^{2}} d_{x}-U_{0} \pi D_{e v} d_{x}\left(T_{c}-T_{a m b}\right)=\rho_{0} C_{p} A_{c} d_{x} \frac{\partial T}{\partial t}
$$

Dividiendo todo para $k A_{\bullet}$ :

$\frac{\partial^{2} T}{\partial x^{2}}-\frac{U_{0} \pi D_{e v}\left(T_{c}-T_{a m b}\right)}{k A_{c}}=\frac{\rho_{c} C_{y}}{K} \frac{\partial T}{\partial t}$

El término $\frac{k}{p_{\varepsilon} c_{\mathrm{p}}}$ depende de las propiedades físicas del material y representan la difusividad térmica $\alpha$

Reemplazando:

$\left(T_{0}-T_{a m b}\right)=\theta$

$\frac{\partial^{2} \theta}{\partial x^{2}}-\frac{U_{0} \pi D_{e v} \theta}{k A_{\theta}}=\frac{1}{\alpha} \frac{\partial T}{\partial t}$

En el estado estacionario no hay variación de temperatura con respecto al tiempo, por la tanto la expresión es la siguiente:

$\frac{\partial^{2} \theta}{\partial x^{2}}-\frac{U_{0} \pi D_{\theta c} \theta}{k A_{0}}=0$ 
Resolviendo esta ecuación diferencial de segundo orden y aplicando condiciones de contorno cuando $x=L$ y $T=T_{L}$ se obtiene:

Figura 6. Condiciones de contorno

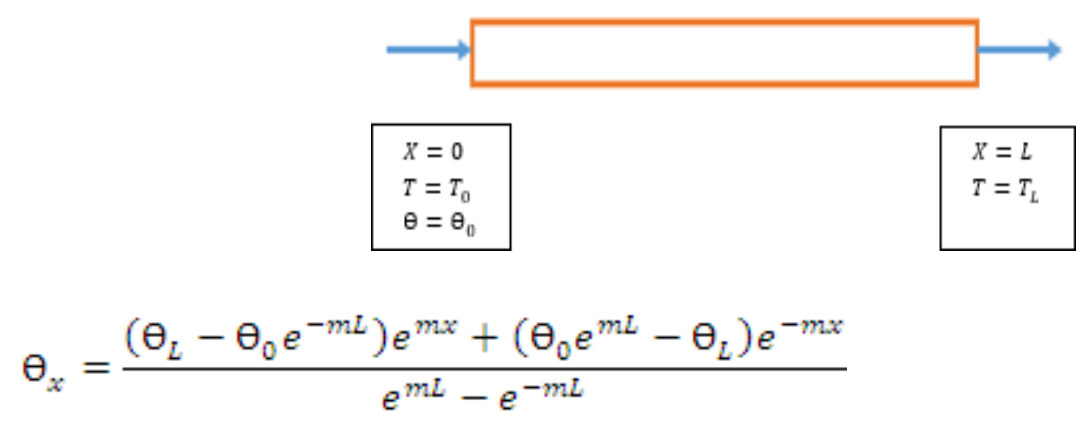

Con esta expresión se encuentra el perfil de temperatura en sentido longitudinal en el tubo cuando se encuentra calentándose en el interior en estado estacionario.

El coeficiente global de transferencia de calor $U_{0}$ se lo obtiene realizando un análisis de resistencias térmicas del tubo absorbedor.

Figura 7. Esquema del tubo absorbedor

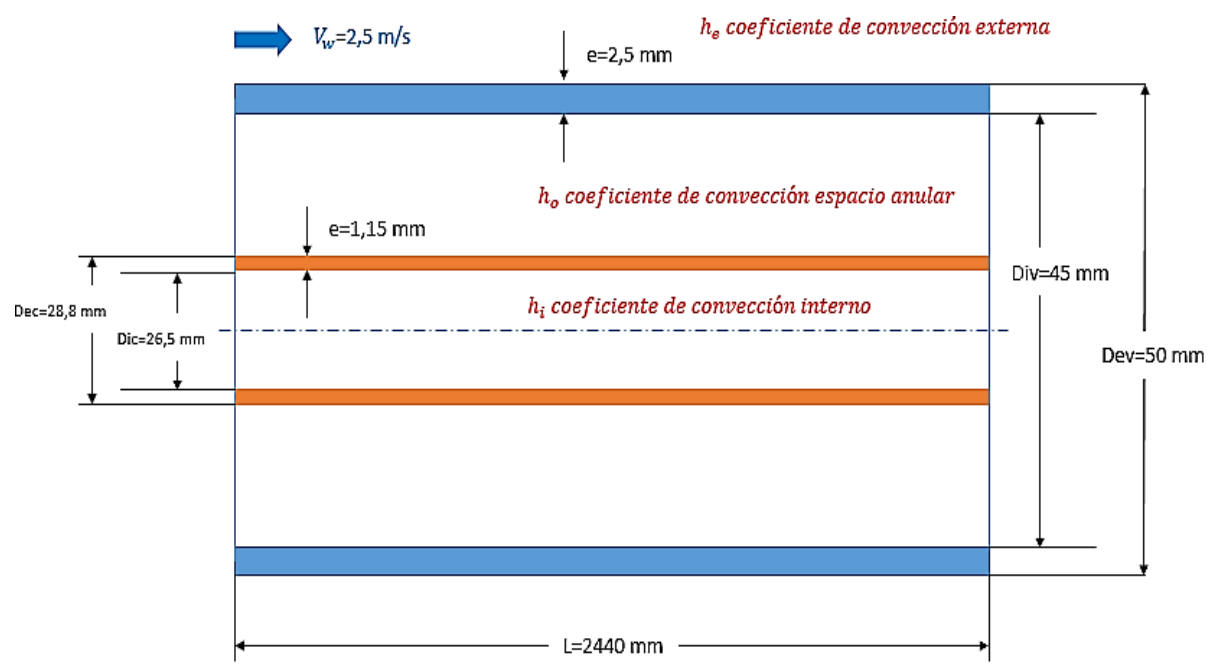

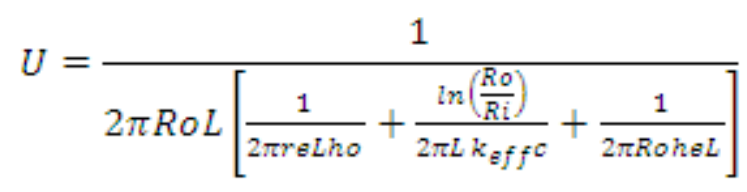

El valor de ho se determina mediante la siguiente expresión: 


$$
\begin{aligned}
& N u_{D}=\frac{h o D}{k} \\
& N u_{D}=(0,55)\left(G r_{L} \mathrm{Pr}\right)^{1 / 4} \\
& G r_{L}=\frac{g \beta\left(T_{s}-T_{\infty}\right) D^{3}}{v^{2}}
\end{aligned}
$$

El valor de he es igual a:

$$
h_{e}=2,8+3 V_{w}
$$

La conductividad térmica efectiva se halla de la siguiente forma:

$$
\begin{aligned}
& \frac{k_{\text {eff }}}{k}=0,396\left[\frac{\ln \left(\frac{D_{0}}{D_{i}}\right)}{b^{\frac{\pi}{4}}\left(\frac{1}{D i^{\frac{3}{4}}}+\frac{1}{D_{0} \frac{3}{4}}\right)^{\frac{5}{4}}}\right]\left(\frac{P r}{0,861+P r}\right)^{\frac{1}{4}} R a_{b}^{\frac{1}{4}} \\
& R a_{b}=G r \cdot P r
\end{aligned}
$$

Todas las propiedades deben ser evaluadas a la temperatura fílmica.

\section{Resultados y discusión}

Establecer las dimensiones adecuadas del calentador solar es una de las partes más importantes del sistema de calentamiento, ya que de éstas depende una correcta captación de rayos solares, además que influye de manera considerable en los resultados del proceso térmico que ocurre en el colector.

Tabla 1. Parámetros geométricos, ópticos y térmicos del colector solar cilíndrico parabólico

\begin{tabular}{|l|c|c|}
\hline \multicolumn{1}{|c|}{ NOMBRE } & Valor & Unidades \\
\hline Ancho del concentrador & 1 & $\mathrm{~m}$ \\
Longitud del concentrador & 2,44 & $\mathrm{~m}$ \\
Diámetro del receptor & 0,0254 & $\mathrm{~m}$ \\
Diámetro de cubierta de vidrio & 0,05 & $\mathrm{~m}$ \\
Área de apertura & 2,3424 & $\mathrm{~m}^{2}$ \\
Área del receptor & 0,1947 & $\mathrm{~m}^{2}$ \\
Factor de concentración & 12,03 & - \\
Altura de la parábola & 0,29 & $\mathrm{~m}$ \\
\hline
\end{tabular}


Distancia focal teórica

Distancia focal experimental

Reflexividad del reflector

Transmisividad de la cubierta de vidrio

Absortividad del tubo de cobre

Coeficiente global de pérdidas de

transferencia de calor
$0,225 \quad \mathrm{~m}$

$0,235 \quad \mathrm{~m}$

0,572 adimensional

0,92 adimensional

0,92 adimensional

10,83

La radiación directa en cualquier lugar del planeta está en función de la hora del día, el número del día del año y la latitud del lugar.

La radiación efectiva que se obtiene en el tubo absorbedor depende de la eficiencia óptica del concentrador.

A continuación se muestra la radiación en la ciudad de Riobamba desde las 9 de la mañana hasta las 4 de la tarde.

Tabla 2. Radiación directa y efectiva en el tubo absorbedor

\begin{tabular}{|c|c|c|}
\hline $\begin{array}{l}\text { Radiación directa } \\
\text { (I) }\left[\mathbf{W} / \mathbf{m}^{2}\right]\end{array}$ & $\begin{array}{c}\text { Rendimiento óptico } \\
{[\text { no }]}\end{array}$ & $\begin{array}{c}\text { Radiación efectiva } \\
{\left[\mathbf{W} / \mathbf{m}^{2}\right]}\end{array}$ \\
\hline 706,44 & 43,7 & 308,71 \\
\hline 792,28 & 43,7 & 346,22 \\
\hline 864,61 & 43,7 & 377,83 \\
\hline 922,19 & 43,7 & 402,99 \\
\hline 964,04 & 43,7 & 421,28 \\
\hline 989,43 & 43,7 & 432,38 \\
\hline 997,95 & 43,7 & 436,1 \\
\hline 989,43 & 43,7 & 432,38 \\
\hline 964,04 & 43,7 & 421,28 \\
\hline 922,17 & 43,7 & 402,99 \\
\hline 864,61 & 43,7 & 377,83 \\
\hline 792,28 & 43,7 & 346,22 \\
\hline 706,44 & 43,7 & 308,71 \\
\hline 608,56 & 43,7 & 265,94 \\
\hline 500,31 & 43,7 & 218,63 \\
\hline
\end{tabular}

Fuente: (Autores) 


\section{Resultados experimentales y teóricos del tiempo de calentamiento de agua hasta $7^{\circ} \mathrm{C}$}

Para realizar las pruebas experimentales se tomaron datos del tiempo que demora en calentarse el agua dentro del tubo absorbedor hasta $70^{\circ} \mathrm{C}$ mientras permanece el fluido estancado. Esta prueba se la realizó durante el mes de noviembre de 2016 desde las 9 de la mañana hasta las 4 de la tarde, en intervalos de 30 minutos.

Tabla 3. Tiempo de calentamiento de agua hasta $70^{\circ} \mathrm{C}$ a diferentes horas del día

\begin{tabular}{|c|c|c|c|}
\hline Hora & $\begin{array}{c}\text { Calor Útil } \\
{[\mathbf{W}]}\end{array}$ & $\begin{array}{c}\text { Tiempo teórico } \\
\text { [seg] }\end{array}$ & $\begin{array}{c}\text { Tiempo experimental } \\
\text { [seg] }\end{array}$ \\
\hline $9: 00$ & 510,7 & 707 & 750,8 \\
$9: 30$ & 591,3 & 605,5 & 644,3 \\
$10: 00$ & 660,7 & 537,4 & 560,2 \\
$10: 30$ & 715,2 & 492,2 & 510,2 \\
$11: 00$ & 756,7 & 461,2 & 467,2 \\
$11: 30$ & 780,6 & 431,7 & 434,6 \\
$12: 00$ & 791,3 & 418,3 & 420,1 \\
$12: 30$ & 783,9 & 422,2 & 425,3 \\
$13: 00$ & 758,7 & 440,2 & 444,7 \\
$13: 30$ & 718,5 & 473,2 & 478,3 \\
$14: 00$ & 664 & 516,6 & 530,2 \\
$14: 30$ & 594,7 & 586,9 & 598 \\
$15: 00$ & 514 & 684,9 & 691,9 \\
$15: 30$ & 422,5 & 840,3 & 867,2 \\
$16: 00$ & 321,4 & 1123 & 1139,8 \\
\hline
\end{tabular}

Figura 8. Tiempo de calentamiento de agua hasta $70^{\circ} \mathrm{C}$ a diferentes horas del día

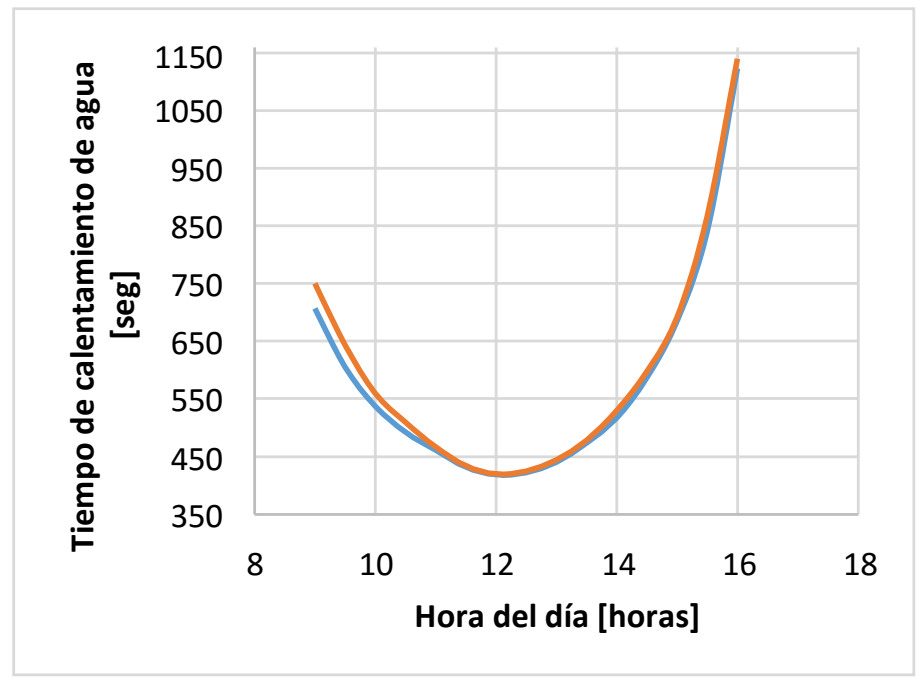


Como era de esperarse, el tiempo de calentamiento de agua es menor a medida que la radiación aumenta, la gráfica muestra que aproximadamente desde las 11 am hasta las 13:30 pm el tiempo que demora en calentarse el agua es bajo, a partir de esa hora el tiempo vuelve a aumentar debido a que la radiación empieza a disminuir.

\section{Resultados del incremento de temperatura del agua a diferentes velocidades}

El caudal medido dentro del sistema es de 0,25 L/s. A partir de ese caudal y del área del tubo se calculó las diferentes velocidades con las que se experimentó.

Tabla 4. Incremento de temperatura a diferentes velocidades de fluido

\begin{tabular}{|c|c|c|}
\hline Velocidad $(\mathbf{m} / \mathbf{s})$ & $\begin{array}{c}\text { Incremento } \\
\mathbf{d e} \\
\text { temperatura } \\
\text { teórico }\left({ }^{\circ} \mathbf{C}\right)\end{array}$ & $\begin{array}{c}\text { Incremento de } \\
\text { temperatura } \\
\text { experimental }\left({ }^{\circ} \mathbf{C}\right)\end{array}$ \\
\hline 0,12 & 3,45 & 3,4 \\
0,102 & 4,049 & 4,2 \\
0,082 & 5,017 & 4,9 \\
0,049 & 8,253 & 8,4 \\
0,034 & 11,53 & 11,6 \\
\hline
\end{tabular}

Figura 9. Incremento de temperatura a diferentes velocidades de fluido

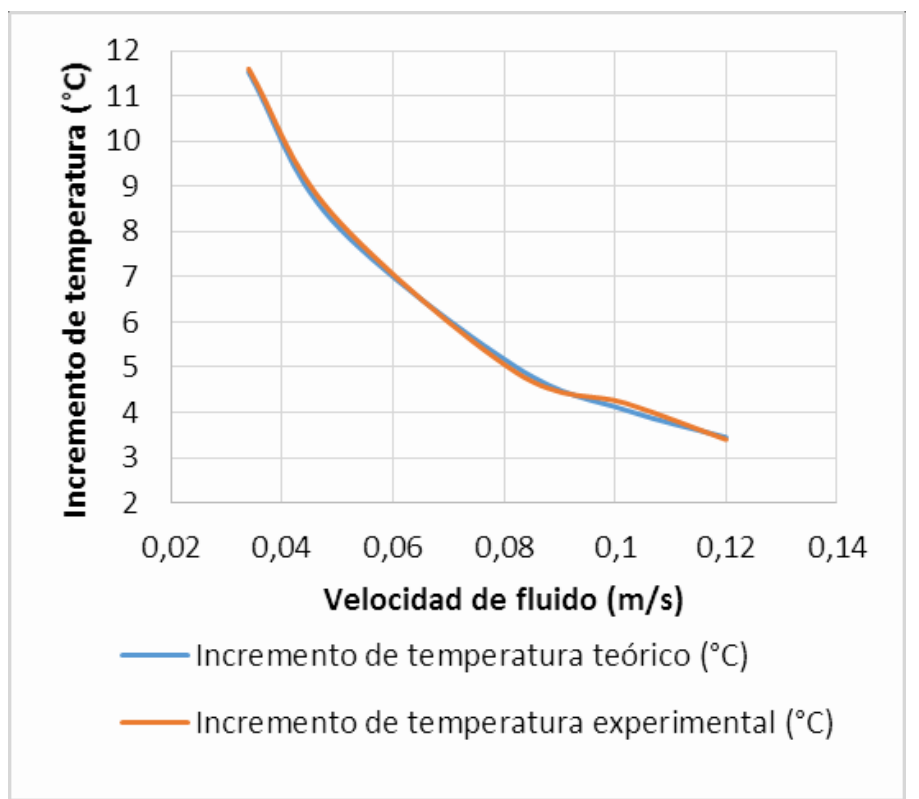


Como se observa en la figura mientras el fluido pasa a través de la tubería a una velocidad muy baja, adquiere mayor temperatura, mientras la velocidad sigue aumentado el incremento de temperatura en el fluido disminuye. Por lo tanto como conclusión es que a menor velocidad de fluido mayor es el incremento en la temperatura.

\section{Resultados del perfil de temperatura en el tubo absorbedor en régimen estacionario}

El perfil que se obtiene a partir de la ecuación del gradiente de temperaturas en sentido longitudinal. Esta ecuación indica el calentamiento lineal del agua y es una función de la longitud del tubo receptor de $2,44 \mathrm{~m}$, por esta razón se ha dividido al tubo en intervalos de 0,3 $\mathrm{m}$ para apreciar de mejor manera la tendencia de la curva. La máxima temperatura que alcanza el fluido es de $70^{\circ} \mathrm{C}$.

Tabla 5. Resultado perfil de temperatura a diferentes distancias

\begin{tabular}{|c|c|}
\hline $\mathbf{L}$ [m] & $\boldsymbol{\theta}\left[{ }^{\circ} \mathbf{C}\right]$ \\
\hline 0,3 & 56,7251262 \\
0,6 & 55,0632486 \\
0,9 & 54,4579657 \\
1,2 & 54,1867693 \\
1,5 & 54,0586807 \\
1,8 & 53,9954274 \\
2,1 & 53,9618426 \\
2,4 & 53,9418558 \\
\hline
\end{tabular}

Esta tabla muestra el comportamiento de la temperatura dentro del tubo absorbedor a diferentes distancias de longitud de tubería.

Figura 10. Gradientes de temperatura en función de la longitud del tubo en régimen estacionario

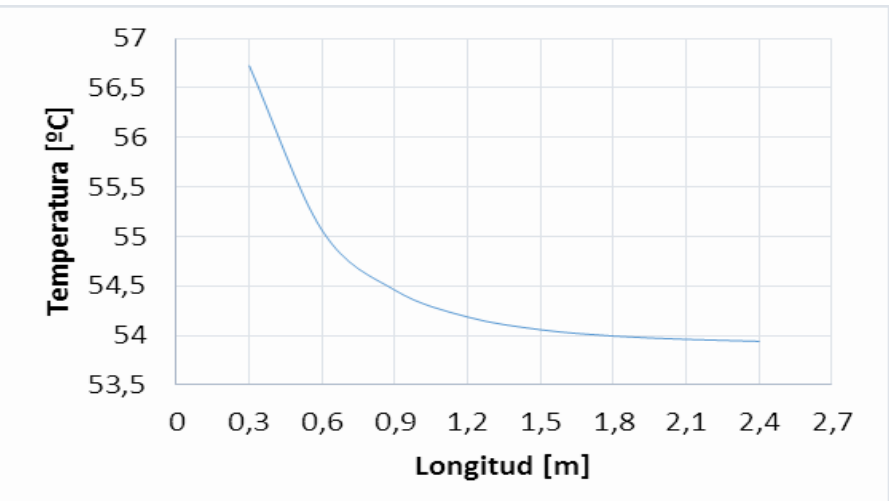


Comparación de la eficiencia máxima y eficiencia promedio entre el colector solar de tubos al vacío y el colector solar cilíndrico parabólico.

La eficiencia del calentador cilíndrico parabólico depende del calor útil, del área de apertura y de la radiación directa solar.

Tabla 6. Eficiencia del colector solar cilíndrico parabólico durante las diferentes horas del día

\begin{tabular}{|c|c|c|c|}
\hline Hora & $\begin{array}{c}\text { Radiación } \\
{\left[\mathbf{W} / \mathbf{m}^{2}\right]}\end{array}$ & $\begin{array}{c}\text { Calor útil } \\
{[\mathbf{W}]}\end{array}$ & $\begin{array}{c}\text { Eficiencia } \\
{[\%]}\end{array}$ \\
\hline $9: 00$ & 706 & 540,9 & 33,0520841 \\
9.30 & 792 & 591,3 & 33,2084085 \\
$10: 00$ & 864 & 689,9 & 34,4476001 \\
$10: 30$ & 922 & 747,4 & 34,9710555 \\
$11: 00$ & 964 & 791 & 35,3985944 \\
$11: 30$ & 989 & 817,2 & 35,6466428 \\
$12: 00$ & 997 & 828,5 & 35,8495677 \\
$12: 30$ & 989 & 820,2 & 35,7775042 \\
$13: 00$ & 964 & 795,7 & 35,6089274 \\
$13: 30$ & 922 & 754,2 & 35,2892294 \\
$14: 00$ & 864 & 697,7 & 34,8370642 \\
$14: 30$ & 792 & 627 & 34,1530055 \\
$15: 00$ & 706 & 544,5 & 33,2720647 \\
$15: 30$ & 608 & 453,4 & 32,1709959 \\
$16: 00$ & 500 & 350,6 & 30,2502157 \\
\hline
\end{tabular}

Se observa que la máxima eficiencia es de 35,84 \% al medio día, y una eficiencia promedio de $34,26 \%$.

La eficiencia máxima de un colector solar de tubos al vacío con una inclinación de $45^{\circ}$ se muestra en la siguiente tabla obtenida del trabajo de Bérriz Peréz:

Tabla 7. Eficiencia energética de colector con tubos al vacío

\begin{tabular}{|l|c|c|}
\hline Horario & $\begin{array}{c}\text { Radiación } \\
\text { Solar } \\
{\left[\mathbf{k C a l} / \mathbf{m}^{2}\right]}\end{array}$ & Eficiencia\% \\
\hline 9:00-10:00 & 400 & 60 \\
10:00-11:00 & 450 & 64 \\
11:00-12:00 & 480 & 67
\end{tabular}




\begin{tabular}{|ccc|} 
12:00-13:00 & 640 & 72 \\
13:00-14:00 & 600 & 74 \\
14:00-15:00 & 560 & 71 \\
15:00-16:00 & 450 & 66 \\
TOTAL & $\mathbf{3 5 8 0}$ & $\mathbf{6 8}$ \\
\hline
\end{tabular}

Se observa que la máxima eficiencia del colector es de $74 \%$ y se produce a la 1 de la tarde. A continuación en la siguiente figura se muestra la comparación entre ambos colectores.

Figura 11. Comparación de la eficiencia del calentador solar cilíndrico parabólico y del colector solar de tubos al vacío durante las diferentes horas del día

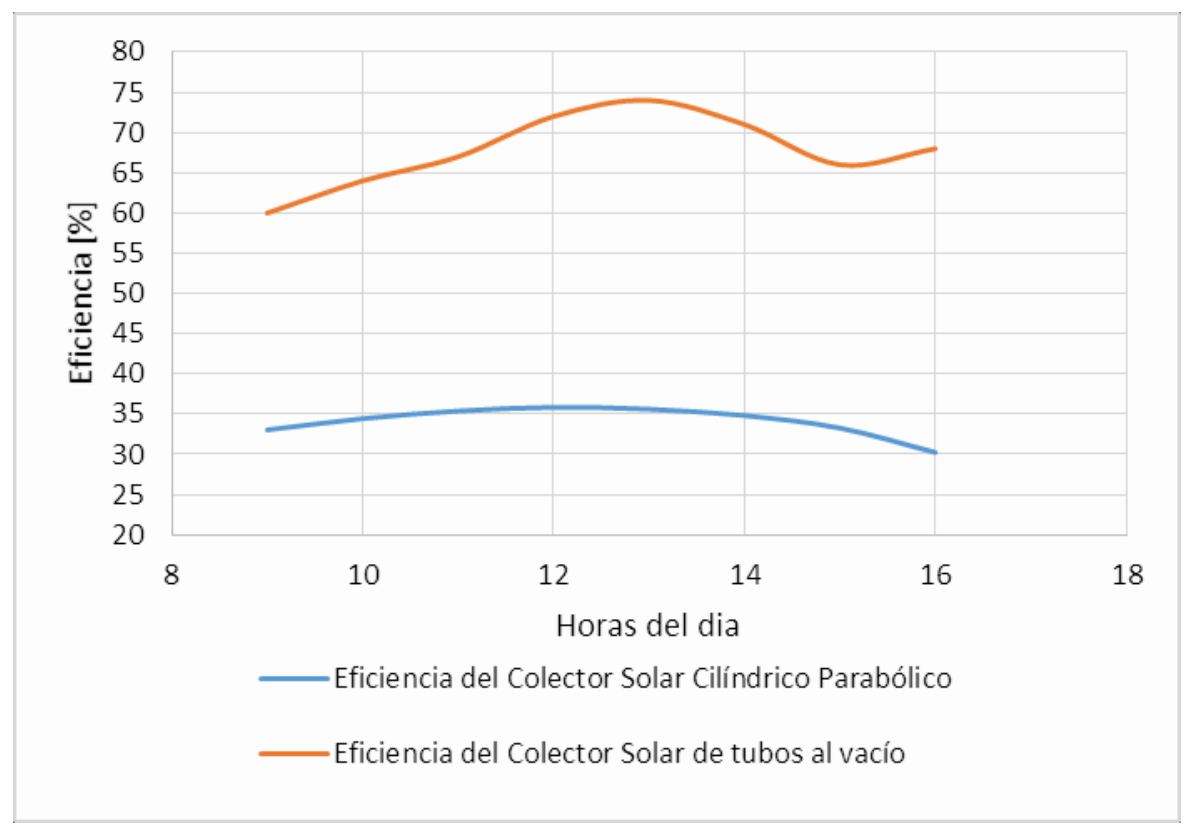

La eficiencia térmica máxima del colector solar de tubos al vacío es mayor con un valor de $74 \%$, mientras que la eficiencia máxima del colector solar cilíndrico parabólico es de 35,84\%. Esto es debido a que el tubo del primer colector por encontrarse al vacío reduce significativamente las pérdidas térmicas lo cual ayuda a mejorar la eficiencia.

El calentador solar cilíndrico parabólico calienta en menor tiempo el agua dentro de un tubo debido a la concentración solar, pero menor cantidad de volumen debido a que se posee un solo tubo, a diferencia de un colector de tubos al vació que posee varios tubos. 
La mayor ganancia de calor en el tubo absorbedor se da en sentido radial. El análisis del presente trabajo se lo realizo en sentido longitudinal en el cual no se considera la distribución térmica en el área efectiva. Para facilitar el cálculo se toma en cuenta un sistema ideal, en el cual se considera un equilibrio térmico, lo que significa que el agua está a la misma temperatura que la tubería de cobre.

\section{Conclusiones}

El volumen de agua a calentar mediante este sistema es una media de 7 litros por hora, debido a que el sistema entra en funcionamiento desde las 9:00 am y termina su proceso a las 4:00 pm, se puede llegar a obtener 50 litros al día, de agua caliente. A partir de las 4 de la tarde el sistema de seguimiento vuelve a su posición inicial, sin embargo hay que tener en cuenta que existe radiación en todo momento, por lo tanto esa radiación existente después de las 4 pm puede ser aprovechada para mantener al sistema de calentamiento estable o para evitar una disminución de temperatura en el colector.

La modelación matemática permitió encontrar una ecuación que determina el gradiente de temperatura en estado estacionario. El perfil de temperatura en sentido longitudinal del tubo absorbedor es de tipo exponencial.

Se demostró que a mayor velocidad existe menor incremento de temperatura, se logró variando la apertura de la válvula, de un $20 \%$ de apertura a una velocidad de $0,034 \mathrm{~m} / \mathrm{s}$, se consigue un incremento de $11,6^{\circ} \mathrm{C}$, y con un $100 \%$ de apertura a una velocidad de $0,12 \mathrm{~m} / \mathrm{s}$ se consiguió un incremento de $3,4{ }^{\circ} \mathrm{C}$. Por lo tanto el agua presenta un menor incremento de temperatura a mayor velocidad debido a que al fluir de manera rápida está menos tiempo en contacto con el tubo absorbedor.

La ubicación del punto focal teórico no coincide con el punto focal experimental, debido a imperfecciones de construcción en el reflector solar, es importante ubicar el tubo absorbedor de manera experimental, ya que una mala posición no permite que haya una correcta incidencia de rayos solares en el tubo y por lo tanto el agua no alcanza la temperatura deseada. 
A diferencia de un colector solar sin concentración (tubos al vacío), el colector solar cilíndrico parabólico debe hacer un seguimiento exacto de Este a Oeste, y ubicado de Norte a Sur, para que los rayos solares siempre estén en dirección al punto focal.

La eficiencia del calentador solar cilíndrico parabólico en comparación con el colector solar de un tubo al vacío es menor, debido a que las pérdidas térmicas en el sistema de calentamiento del colector cilíndrico parabólico son mayores, ya que el espacio anular entre la cubierta de vidrio y el tubo de cobre no se encuentra al vacío, a diferencia del tubo al vacío en el que se reducen significativamente las pérdidas. El sistema que brinda el mayor volumen de agua es el colector de tubos al vacío debido a que el área de captación solar es mucho mayor que el área del colector cilíndrico parabólico, es decir que se tiene una mayor cantidad de tubos.

Debido a la concentración de rayos solares en la línea focal en el tubo absorbedor la ganancia de calor útil es mayor a la de un tubo al vacío. Esto se debe a que el área de apertura del colector cilíndrico parabólico es mayor al área de apertura de un tubo al vacío.

\section{Referencias Bibliográficas}

BARAGOZZA BASTIDAS, Edison \& RISCO DE LEÓN, Juan. Diseño de un Colector Solar Cilíndrico Parabólico y Construcción de un Prototipo. [En línea] Corporacióm Umiversitaria Tecomologica del Volivar, Facultad de Mecámica. Cartagena, Colombia. 2002. pp. 177-179. [Consulta: 14 diciembre 2016]. Disponible en: biblioteca.unitecnologica.edu.co/notas/tesis/0001057.pdf

BENAVIDES SALCEDO, Enrique Santiago. Diseño y construcción de una cocina solar experimental de tipo cilíndrico parabólico con aplicación al sector rural marginal. Escuela Superior Politécmica del Ejercito, Sangolquí, Ecuador. 2006. pp 80-84

CABANA, Wilson, et al. Análisis del comportamiento térmico de un tubo de vacío para el calentamiento de agua . [En línea] Arequipa. [Consulta: 14 de diciembre 2016]. Disponible en: http://perusolar.org/16spesponencias/ANALISIS\%20DEL\%20COMPORTAMIENTO\%20TER MICO\%20DE\%20UN\%20TUBO\%20DE\%20VACIO\%20PARA\%20EL\%20CALENTAMIENT O\%20DE\%20AGUA.ppt. 
CENGEL, Yunus. Termodinàmica. 3a ed. Mexico : Mc Graw Hill, 2010. pp. 17 - 27.

CENGEL, Yunus \& GHAJAR, Afshin. Transferencia de Calor. 4a ed. Mexico, McGraw-Hill, 2011. pp. $700-708$.

ECHAZÚ, CADENA \& SARAVIA. Estudio de Materiales Reflectivos para Concetradores Solares. [En línea]. Buenos Aires, Argemtima. [Consulta: 14 de diciembre 2016]. Disponible en: http://www.cricyt.edu.ar/asades/modulos/averma/trabajos/2000/2000-t008-a003.pdf

ECHEVERRÍA LÓPEZ, César Antonio. Diseño de un colector cilindrico parabólico compuesto con aplicación para el calentamiento de agua. Umiversidad de Piura. Facultad de Imgemiería. Piura, Perú. 87. 2011.pp Disponible https://pirhua.udep.edu.pe/bitstream/handle/11042/1389/IME_159.pdf.

ENERGÍA SOLAR. Energía Solar. [En línea] .2015. [Consulta: 19 diciembre 2016]. Disponible en: https://solar-energia.net/energia-solar-termica/alta-temperatura.

HERNÁNDEZ ECHEVERRÍA, Yoshito. Diseño de un concentrado solar de geometría paraboidal portátil .Umiversidad tecmológica de la Mixteca. Oaxaca, Mexico. 2012. pp 24

HOLMAN, J.P. Transferencia de Calor. 8va ed. Madrid, España. McGrawHill. 1999. pp. 5.

HOM, Manfred, RODRIGUEZ, Juan \& VEGA, Patricia. Promoviemdo la utilizacióm de la emergia solar em la Provimcia de Espimar como altermativa amviemtal para el homvre. Cusco, Perú, 2004. pp 3

IBARRA SALAZAR, Byron Alejando \& ROJAS PACHECO, Franklin Javier. Diseño y construcción de un prototipo de colector solar parabólico giratorio de mediana temperatura. Quito, Ecuador. 2012. pp 44

INCROPERA, Frank \& DEWITT, David. Fundamentos de Transferencia de Calor . Mexico. Prentice Hall. 1990. pp 70

JIMÉNEZ ARIAS , Hernán Santiago \& PORTALANZA MOLINA, Narcisa. Estandarización de Sistemas de calentamiento de agua con Energía Solar en la ciudad de Riobamba. [En linea] . 
Escuela Superior Politécmica de Chimvorazo. Facultad de Mecámica. Riobamba, Ecuador.2009. pp. 32. Disponible en: http://dspace.espoch.edu.ec/bitstream/123456789/269/1/15T00417.pdf

LLORENTE SANCHÉZ, Irene. Estado del arte de tubos receptores para captadores cilíndrico parabólicos. Comparación de modelos estáticos.[En linea]. Umiversidad de Sevilla, Escuela Técmica Superir de Imgemiería. 2015. [Consulta 19 de diciembre 2016]. pp 33-40. Disponible en:

http://bibing.us.es/proyectos/abreproy/5652/fichero/PROYECTO+FIN+DE+CARRERA.+Irene+ Llorente.pdf

McCORMAC, Jack y CSERNAK, Stephen. Diseño de estructuras de acero. 5a ed. Mexico. Alfaomega. 2012. pp. 42.

ORBEA SAMANIEGO, Edison Gustavo \& SILVA BRAVO, Jorge Luis. Ingeniería inversa y repotenciación para la optimización, toma de datos y control del colector cilíndrico parabólico de 7,2 m2. Escuela Superior Politécmica del Ejercito. Facultad de Mecámica. Sangolquí, Ecuador. 2014. pp. 80.

PAREDES , Carmen. Diseño de Captador Solar Cilindrico Parabólico para aplicaciones Rurales en Paraguay. [En linea] Escuela Técmica Superior de Imgemieros de Mimas. 2012. pp. 31-34. Disponible en: http://oa.upm.es/14011/1/PFC_CARMEN_PAREDES_VELASCO.pdf.

SERRANO GARCÍA, Daniel. Cálculo, Diseño y Análisis de una central termosolar de Colectores Cilíndricos-Parabólicos operando con Sales Fundidas. Leganés, España. 2012. pp.40. 